\title{
PERFORMANCE ANALYSIS OF WDM OPTICAL NETWORKS WITH WAVELENGTH USAGE CONSTRAINT *
}

\author{
Jing Fang, R.Srinivasan and Arun K.Somani \\ Dependable Computing \& Networking Laboratory \\ Department of Electrical and Computer Engineering \\ Iowa State University, Ames, IA 50011 \\ jiang@iastate.edu, rsini@iastate.edu, arun@iastate.edu
}

\begin{abstract}
Due to power considerations, it is possible that not all wavelengths available in a fiber can be used at a given time. In this paper, an analytical model is proposed to evaluate the blocking performance of wavelength-routed optical networks with and without wavelength conversion where the usable wavelengths in a fiber is limited to a certain maximum number, referred to as wavelength usage constraint. The effect of the wavelength usage constraint is studied on ring and mesh-torus networks. It is shown that the analytical model closely approximates the simulation results. It is observed that increasing the total number of wavelengths in a fiber is an attractive alternative to wavelength conversion when the number of usable wavelengths in a fiber is maintained the same.
\end{abstract}

Keywords: Optical networks, Wavelength usage constraint, Blocking probability

\section{Introduction}

Wavelength Division Multiplexing (WDM) has emerged as the promising technology to meet the ever-increasing demand for bandwidth. WDM divides the available fiber bandwidth into WDM channels, called wavelengths, each operating at peak electronic rate. Connections between users are supported by establishing an all-optical channel between the end nodes. The all-optical connections are referred to as lightpaths. Wavelength converter is a device that allows the optical signal on a wavelength to be converted into another wavelength. In the absence of wavelength converters, a lightpath should oc-

* The research reported in this paper is funded in part by the National Science Foundation under grant ANI-9973102 and Defense Advanced Research Projects Agency and National Security Agency under grant N66001-00-1-8949. 
cupy the same wavelength on all the links it traverses. This property is known as the wavelength-continuity constraint. Hence a connection request encounters higher blocking probability in a network without wavelength conversion than it does in a network that employ full-wavelength conversion at all nodes. Although wavelength converters improve network blocking performance, the high cost of wavelength converters have made it impractical to employ fullwavelength converters at all nodes.

The role of wavelength converters in wavelength-routed networks has been studied extensively in the literature $[1,3,4,5,6,10]$. The role of sparsewavelength conversion, where only a few nodes in the network have fullwavelength conversion capability, has been analyzed in [10]. The effect of limited-wavelength conversion, where a given input wavelength can be converted into a set of (but not all) output wavelengths, has been studied in [8] and [11]. Multi-fiber multi-wavelength wavelength-routed networks have been shown to offer blocking performance similar to that of networks that employ limited- or sparse-wavelength conversion [7, 12, 13]. A generalized framework for analyzing optical networks that employ both wavelength and time division multiplexing has been recently proposed in [9] from which most of the models discussed above can be derived.

The available fiber bandwidth can be divided into a large number of wavelengths while maintaining the operating speed of each wavelength to be around the peak electronic speeds. However, due to power constraints not all the wavelengths can be used at any given instant in time. This restriction is referred to as the wavelength usage constraint. Such a scenario could arise due to the restriction on the power carried in the fiber or the power limit on the optical components on the path such as amplifiers, re-generators, etc. Two alternatives can be employed to solve this problem: (1) Employing wavelength converters with the number of wavelengths carried in the fiber being the same as the maximum number of usable wavelengths; or (2) Employing more wavelengths in a fiber but restricting the number of usable wavelengths to a certain maximum without employing wavelength converters.

We illustrate the wavelength usage constraint and motivate the two solutions through a simple example. Consider a two-link path of a network as shown in Figure 1(a). The nodes are assumed to be connected by a single-fiber link. Each fiber is assumed to carry signals in at-most two wavelengths at any given time due to power considerations. Assume that two calls are established in the path; the first call originating from Node 1 and destined for Node 2 established on wavelength $W_{1}$ and second call originating from Node 2 and destined for Node 3 established on wavelength $W_{2}$.

Consider a third call that originates from Node 1 destined for Node 3. If the fibers were to carry only two wavelengths and wavelength conversion is not employed, then the third call would be blocked. The third call would be 


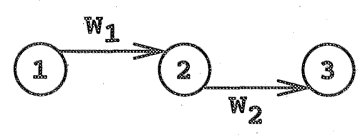

(a)

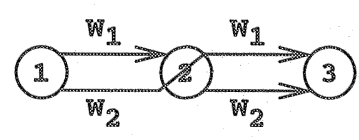

(b)

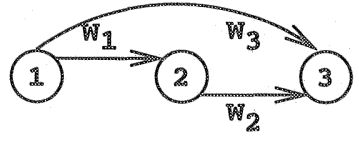

(c)

Figure 1. A two-link network. (a) 2 wavelengths per fiber with no wavelength conversion. (b) 2 wavelengths per fiber with wavelength conversion. (c) 3 wavelengths per fiber with at-most 2 usable at any given time and no wavelength conversion.

accepted if wavelength conversion is employed. A connection can be established from Node 1 to Node 3 by assigning wavelength $W_{1}$ on the first link and wavelength $W_{2}$ on the second, making use of the wavelength conversion capability at Node 2 as shown in Figure 1(b). An alternative to employing wavelength conversion is to increase the number of wavelengths available in a fiber. For example, if the fibers were to carry 3 wavelengths of which only two can be used at any given time, then the third call could be accommodated on wavelength $W_{3}$ without employing wavelength conversion as shown in Figure 1(c).

The goal of this paper is to develop an analytical model for evaluating the blocking performance of wavelength-routed optical network with wavelength usage constraint. The analytical model will then be used to evaluate the above two alternatives. The remainder of the paper is organized as follows: Section 2 provides the network model and assumptions that are used in developing the analytical model. The analytical model for computing the blocking performance of networks with wavelength usage constraint is developed in Section 3. Section 4 compares the analytical and simulation results and discusses the performance of the two alternatives on ring and mesh-torus networks. Section 5 concludes the paper.

\section{Network Model and Assumptions}

A wavelength-routed optical network with $N$ nodes is considered in this paper. The nodes are connected by single fiber links. Each fiber is assumed to carry a total of $W$ wavelengths of which at-most $U$ wavelengths can be used at any given time $(W \geq U)$.

The analytical model developed in this paper is based on the following assumptions:

- Call requests arrive at each node according to a Poisson process with rate $\lambda_{n}$. Each call is equally likely to be destined to any of the remaining nodes. 
The holding time of calls are exponentially distributed with mean $1 / \mu$. The Erlang load offered by a node is $\rho=\lambda_{n} / \mu$.

m The bandwidth requirement of calls are assumed to be of one wavelength capacity.

No broadcast or multicast traffic is considered.

The routing of calls follow fixed-path routing strategy. e.g., shortest-path routing. Although dynamic routing algorithms provide slightly better performance, it is much harder to study them analytically.

n The wavelength assigned for a connection is assumed to be chosen at random from the set of available wavelengths.

- The load on a link of a path is assumed to be correlated only to the load in the previous link of the path, referred to as the Markovian Correlation.

- Blocked calls are discarded and are not re-attempted.

\section{Analysis}

In this section, we develop an analytical model that has modest computation requirements and improves upon the previously proposed Trunk Switched Network models of [9] by restricting the number of usable wavelengths in a fiber.

The network blocking probability is computed as the average blocking probability experienced over different path lengths. Consider a $z$-link path model as shown in Figure 2.

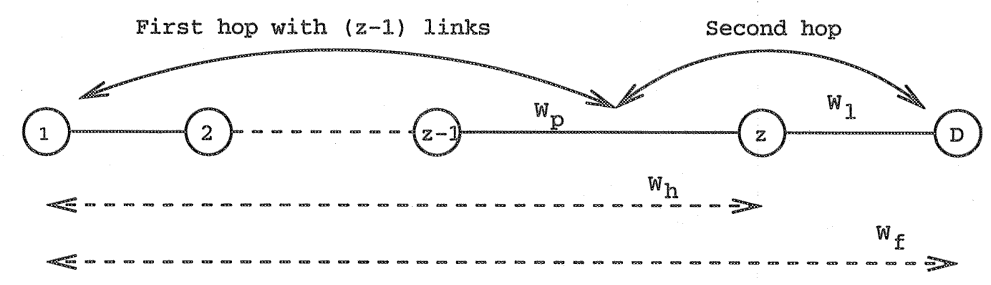

Figure 2. A z-link path model

Let $P_{z}\left(W_{f}\right)$ denote the probability of $W_{f}$ wavelength continuous path available on the $z$-link path. The network blocking probability, denoted by $P_{b}$, is given by:

$$
P_{b}=\sum_{z=1}^{N-1} P_{z}\left(W_{f}=0\right) p_{z}
$$


where $p_{z}$ is the probability of selecting a $z$-link path. The probability of choosing a path of a certain hop length can be computed based on the network topology and routing algorithm employed. $P_{z}\left(W_{f}=0\right)$ denotes the blocking probability over the $z$-link path.

Let $W_{l}$ denote the number of wavelengths free on the last link of the path. Let $P_{z}\left(W_{f}, W_{l}\right)$ denote the probability of $W_{f}$ wavelengths being available on a $z$-link path with $W_{l}$ wavelengths free on the last link. $P_{z}\left(W_{f}\right)$ is then written as:

$$
P_{z}\left(W_{f}\right)=\sum_{W_{l}=W_{f}}^{W} P_{z}\left(W_{f}, W_{l}\right)
$$

The $z$-link path is analyzed as a two-hop path by viewing the first $z-1$ links as the first hop and the last two links as the second hop, as shown in Figure 2. It is to be noted that the destination node is not considered as the last node of the path. Let $W_{h}$ and $W_{p}$ denote the number of wavelengths available on the first hop and the number wavelengths free on the last link of the first hop (link $z-1) . P_{z}\left(W_{f}, W_{l}\right)$ is recursively computed as:

$$
P_{z}\left(W_{f}, W_{l}\right)=\sum_{W_{h}=W_{f}}^{W} \sum_{W_{p}=0}^{W} P_{z-1}\left(W_{h}, W_{p}\right) P\left(W_{f}, W_{l} \mid W_{h}, W_{p}\right)
$$

The starting point of this recursion, for $z=1$, is defined as:

$$
P_{1}\left(W_{f}, W_{l}\right)= \begin{cases}P(W-U) & \text { if } W_{f}=0 \\ P\left(W_{l}\right) & \text { if } W_{f}=W_{l} \text { and } W_{l}>W-U \\ 0 & \text { otherwise }\end{cases}
$$

where $P\left(W_{l}\right)$ denotes the probability of $W_{l}$ free wavelengths on a link. $P\left(W_{l}\right)$ is computed using a two-link path model and is described in Section 3.1.

It is to be noted that when $W_{f}\left(W_{f}>1\right)$ wavelengths are said to be available on a $z$-link path, it is assumed that there is a choice of $W_{f}$ wavelengths on which a call can be established. However, this does not guarantee that $W_{f}$ calls can be accommodated on the $z$-link path as the wavelength usage constraint could be violated at some or all links on the path.

$P\left(W_{f}, W_{l} \mid W_{h}, W_{p}\right)$ is computed by conditioning on the number wavelengths free on the last link as:

$$
P\left(W_{f}, W_{l} \mid W_{h}, W_{p}\right)= \begin{cases}P\left(W_{f} \mid W_{h}, W_{p}, W_{l}\right) P\left(W_{l} \mid W_{h}, W_{p}\right) & \text { if } W_{h} \geq W_{f} \\ 0 & \text { otherwise }\end{cases}
$$

where $P\left(W_{l} \mid W_{h}, W_{p}\right)$ is the probability of $W_{l}$ wavelengths being free on the last link given $W_{h}$ wavelengths are available on the first hop and $W_{p}$ wavelengths free on the last link of the first hop. With the assumption of Markovian 
correlation of link loads, $W_{l}$ is independent of $W_{h}$. Hence, $P\left(W_{l} \mid W_{h}, W_{p}\right)$ is reduced to $P\left(W_{l} \mid W_{p}\right)$. The computation of $P\left(W_{l} \mid W_{p}\right)$ is based on a two-link model and is discussed in section 3.1. Eqn. 5 is rewritten as:

$$
P\left(W_{f}, W_{l} \mid W_{h}, W_{p}\right)= \begin{cases}P\left(W_{f} \mid W_{h}, W_{p}, W_{l}\right) P\left(W_{l} \mid W_{p}\right) & \text { if } W_{h} \geq W_{f} \\ 0 & \text { otherwise }\end{cases}
$$

$P\left(W_{f} \mid W_{h}, W_{p}, W_{l}\right)$ denotes the probability of having $W_{f}$ wavelengths available on a two-link path given that $W_{h}$ wavelengths are free on the first hop with $W_{p}$ wavelengths free on the last link of the first hop and $W_{l}$ wavelengths free on the last link. This probability value is computed by considering two cases: (1) No wavelength conversion and (2) Full-wavelength conversion.

In case 1 , there is no wavelength conversion in the network. Thus wavelength continuity constraint is imposed on the connections. Let $U_{c}$ denote the number of wavelengths that are used by connections that occupy both the links. $P\left(W_{f} \mid W_{h}, W_{p}, W_{l}\right)$ is then obtained as:

$$
P\left(W_{f} \mid W_{h}, W_{p}, W_{l}\right)= \begin{cases}1 & \text { if } W_{f}=0 \text { and }\left(W_{p} \leq W-U \text { or } W_{l} \leq W-U\right) \\
& \sum_{U_{c}=0}^{\min \left(W-W_{p}, W-W_{l}\right)} \frac{\left(\begin{array}{l}
W_{h} \\
W_{f}
\end{array}\right)\left({ }^{W-W_{h}-U_{c}}\right)}{\left(W^{W-W_{c}}\right)} P\left(U_{c} \mid W_{p}, W_{l}\right) \\
& \text { if } W_{f}>0, W_{p}>W-U, W_{l}>W-U, \\
& \text { and } W_{f} \leq \min \left(W_{h}, W_{l}\right) \\
0 & \text { otherwise }\end{cases}
$$

In case 2 , the number of wavelengths available on the path is the minimum number of free wavelengths of each link on the path, provided the total number of used wavelengths in the links are below the maximum value. Thus, a call is blocked only if either or both of the links have $U$ wavelengths occupied. In this case $P\left(W_{f} \mid W_{h}, W_{p}, W_{l}\right)$ is computed as:

$$
P\left(W_{f} \mid W_{h}, W_{p}, W_{l}\right)=\left\{\begin{array}{cl}
1 & \text { if } W_{f}=0, \text { and }\left(W_{p} \leq W-U \text { or } W_{l} \leq W-U\right) \\
1 & \begin{array}{l}
\text { if } W_{f}>0, W_{p}>W-U, W_{l}>W-U \\
\\
\text { and } W_{f}=\min \left(W_{h}, W_{l}\right)
\end{array} \\
0 & \text { otherwise }
\end{array}\right.
$$

The values of $P\left(U_{c} \mid W_{p}, W_{l}\right), P\left(W_{l} \mid W_{p}\right)$, and $P\left(W_{l}\right)$ are obtained using a two-link path model as described in the following subsection. 


\subsection{Free wavelength distribution}

Consider a two-link path model as shown in Figure 3. Let $u_{p}, u_{l}$, and $u_{c}$ denote the number of wavelengths busy on the first link, the number of wavelengths busy on the second link, and the number of wavelengths occupied by calls that continue from the first link to the second, respectively. Note that $u_{c} \leq \min \left(u_{p}, u_{l}\right)$ and $\max \left(u_{p}, u_{l}\right) \leq U$. Recall that at-most $U$ wavelengths out of the total $W$ wavelengths carried by a fiber can be used at any given time.

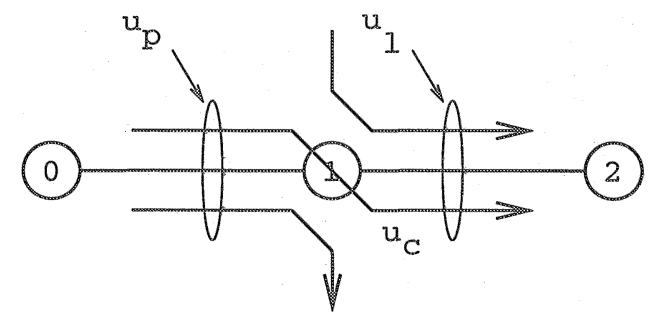

Figure 3. Wavelength occupancy on a 2-hop path.

Let $\lambda_{p}$ denote the arrival rate for calls to the first link, $\lambda_{l}$ denote the arrival rate for calls to the second link, and $\lambda_{c}\left[\lambda_{c} \leq \min \left(\lambda_{p}, \lambda_{l}\right)\right]$ denote the arrival rate of calls to the first link that continue to the second link. If the link loads are assumed to be uniformly distributed, it follows that $\lambda_{p}=\lambda_{l}=\lambda$. The Erlang loads corresponding to the calls that occupy the first link, second link, and that which continue from the first to the second can be written as, $\rho_{p}=\frac{\lambda_{p}}{\mu}$, $\rho_{l}=\frac{\lambda_{l}}{\mu}$, and $\rho_{c}=\frac{\lambda_{c}}{\mu}$, respectively.

The wavelength distribution on a two-link path can be characterized as a 3dimensional Markov chain. The state-space is denoted by the 3-tuple $\left(u_{p}, u_{l}\right.$, $u_{c}$ ). The steady-state probability for the states can be computed as [2]:

$$
\Pi\left(u_{p}, u_{l}, u_{c}\right)=\frac{\frac{\left(\rho_{p}-\rho_{c}\right)^{u_{p}-u_{c}}}{\left(u_{p}-u_{c}\right) !} \frac{\left(\rho_{c}\right)^{u_{c}}}{\left(u_{c}\right) !} \frac{\left(\rho_{l}-\rho_{c}\right)^{u_{l}-u_{c}}}{\left(u_{l}-u_{c}\right) !}}{\sum_{j=0}^{U} \sum_{i=j}^{U} \sum_{k=j}^{U} \frac{\left(\rho_{p}-\rho_{c}\right)^{i-j}}{(i-j) !} \frac{\left(\rho_{c}\right)^{j}}{(j) !} \frac{\left(\rho_{l}-\rho_{c}\right)^{k-j}}{(k-j) !}}
$$

where $0 \leq u_{p} \leq U, 0 \leq u_{l} \leq U, 0 \leq u_{c} \leq \min \left(u_{p}, u_{l}\right)$.

The following probabilities that are required to complete the analytical model are derived from the above steady-state probability.

$P\left(W_{l} \mid W_{p}\right)= \begin{cases}\frac{\sum_{x_{c}=0}^{\min \left(W-W_{l}, W-W_{p}\right)} \Pi\left(W-W_{p}, W-W_{l}, x_{c}\right)}{\sum_{x_{l}=0}^{U} \sum_{x_{c}=0}^{\min \left(W-W_{p}, x_{l}\right)} \Pi\left(W-W_{p}, x_{l}, x_{c}\right)} & \text { for } W-U \leq W_{l}, W_{p} \leq W \\ 0 & \text { otherwise }\end{cases}$ 


$$
\begin{aligned}
& P\left(U_{c} \mid W_{p}, W_{l}\right)=\left\{\begin{array}{cl}
\frac{\Pi\left(W-W_{p}, W-W_{l}, U_{c}\right)}{\sum_{\mathfrak{x}_{c}=0}^{\min \left(W-W_{p}, W-W_{l}\right)} \Pi\left(W-W_{p}, W-W_{l}, x_{c}\right)} \\
& \text { if } U_{c} \leq \min \left(W-W_{p}, W-W_{l}\right) \\
& \text { and } W-U \leq W_{l}, W_{p} \leq W \\
0 & \text { otherwise }
\end{array}\right. \\
& P\left(W_{l}\right)= \begin{cases}\sum_{x_{p}=0}^{U} \sum_{x_{c}=0}^{\min \left(x_{p}, W-W_{l}\right)} \Pi\left(x_{p}, W-W_{l}, x_{c}\right) & \text { for } W-U \leq W_{l} \leq W \\
0 & \text { otherwise }\end{cases}
\end{aligned}
$$

\subsection{Estimation of call arrival rates on a link}

In the analytical model developed in the earlier section, the network traffic was assumed to be known in term of link load. Typically the traffic in the network is specified in terms of set of offered loads between the source and destination node pairs. The call arrival rate has to be estimated from the arrival rates of calls to nodes [10].

Consider a network with $N$ nodes and $L$ links, the average path length of a connection in the network is given by:

$$
Z_{a v}=\sum_{z=1}^{N-1} z p_{z}
$$

where $p_{z}$ is the path-length distribution. Let $\lambda_{n}$ denote the call arrival rate at a node. Let $\lambda$ denote the average link arrival rate and is computed as:

$$
\lambda=\frac{N \lambda_{n} Z_{a v}}{L}
$$

The fraction of traffic that is not destined for a node is obtained as the ratio of the number of links a path that are not the last hop to the total number of links in the path. For a path with $z$ links, there are $(z-1)$ intermediate links. Hence, the fraction of traffic on a link that would continue on any neighboring links at a node is written as:

$$
\begin{aligned}
\delta_{c} & =\frac{\sum_{z=1}^{N-1}(z-1) p_{z}}{\sum_{z=1}^{N-1} z p_{z}} \\
& =1-\frac{1}{Z_{a v}}
\end{aligned}
$$

It is to be noted that the above expression gives the fraction of the traffic that is not destined for a node. Such traffic could continue on any of the output 
links at the node. The link load correlation is defined as the probability that a call on a link would continue to a successive link on a chosen path and is given by:

$$
\gamma_{c}=\left(1-\frac{1}{Z_{a v}}\right) \frac{1}{E}
$$

where $E$ denotes the number of links at a node that do not connect the node to any of the previous nodes in the path, referred to as exit links. Hence, the arrival rate of traffic on a link that would continue to a successive link on a path is given by $\lambda_{c}=\gamma_{c} \lambda$.

\section{Results and Discussion}

In this section, we assess the accuracy of the analytical model by comparing it with the simulation results. Two kinds of network topologies are considered for performance evaluation:

1 a 25 -node bidirectional ring network

2 a $5 \times 5$ bidirectional mesh-torus network

The networks are assumed to employ shortest-path routing. If more than one shortest path is available, one of them is chosen at random. The path length distribution, $p_{z}$, and the number of exit nodes, $E$, for the two networks are given below:

1 Bidirectional Ring network with $N$ nodes (if $N$ is odd):

$$
\begin{gathered}
p_{z}=\frac{2}{N-1} \quad 1 \leq z \leq \frac{N-1}{2} \\
E=1
\end{gathered}
$$

$2 M \times M$ bidirectional mesh-torus network (if $M$ is odd):

$$
\begin{aligned}
& p_{z}= \begin{cases}\frac{4 z}{M^{2}-1} & 1 \leq z \leq \frac{M-1}{2} \\
\frac{4(M-z)}{M^{2}-1} & \frac{M-1}{2}<z \leq M-1\end{cases} \\
& E=3
\end{aligned}
$$

The link load correlation factors for the 25 -node bi-directional ring network and $5 \times 5$ bi-directional mesh-torus network are 0.846 and 0.2 , respectively. 
The selection of these two networks for evaluating the accuracy of the proposed analytical model is due to the high and the low values for link correlation factors. Evaluating the analytical model at these extreme values of link correlation factors would validate the model for a wide range of networks.

For each network, the number of usable wavelengths on each link is fixed as $16(U=16)$. The blocking performance is compared by varying the total number of wavelengths in each fiber. Three different values for the total number of wavelengths in a link are considered: $W=16 ; W=18$, and $W=20$. It is assumed that the networks do not employ wavelength conversion for the above parameters. The blocking performance of networks with the above parameters are compared with that of a network employing 16 wavelengths per fiber and full-wavelength conversion at each node.

Figures 4 and 5 show the blocking performance versus the link load of the two network topologies considered. It is observed that the simulation and the analysis results match closely, thereby validating the analytical model developed in this paper.

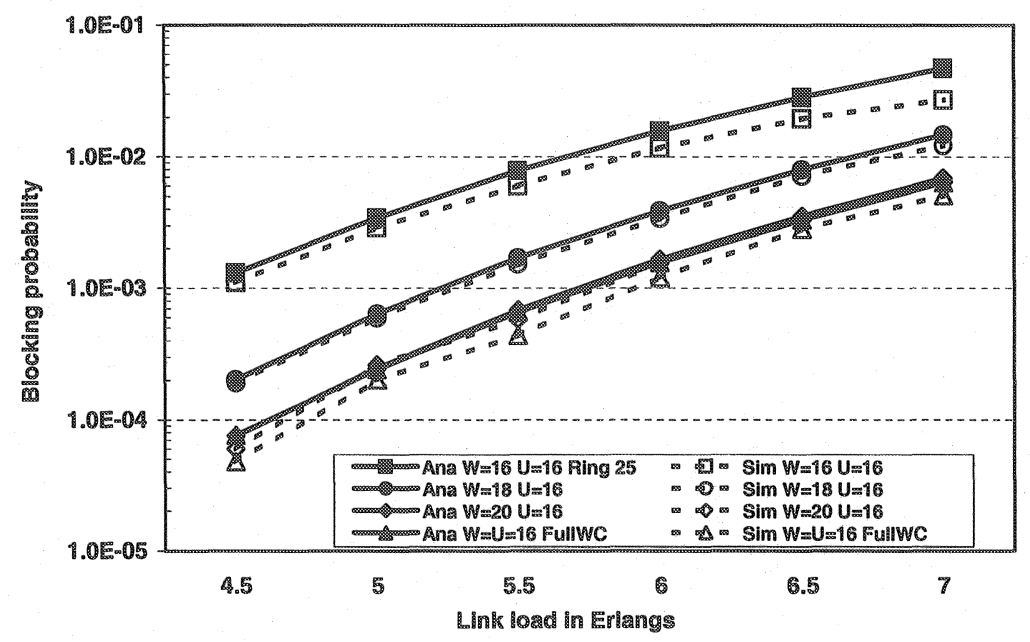

Figure 4. Blocking probability versus the link offered load for a bidirectional ring network with 25 nodes.

It is also observed that there is a significant improvement in the blocking performance when the total number of wavelengths in a link is just a few more than the maximum that can be used at any instant of time. Specifically, for the above parameters, it is observed that a network employing 20 wavelengths per fiber with at-most 16 being usable at any given time has a blocking performance close that of a network employing 16 wavelengths per fiber with full-wavelength conversion capability at all nodes. 


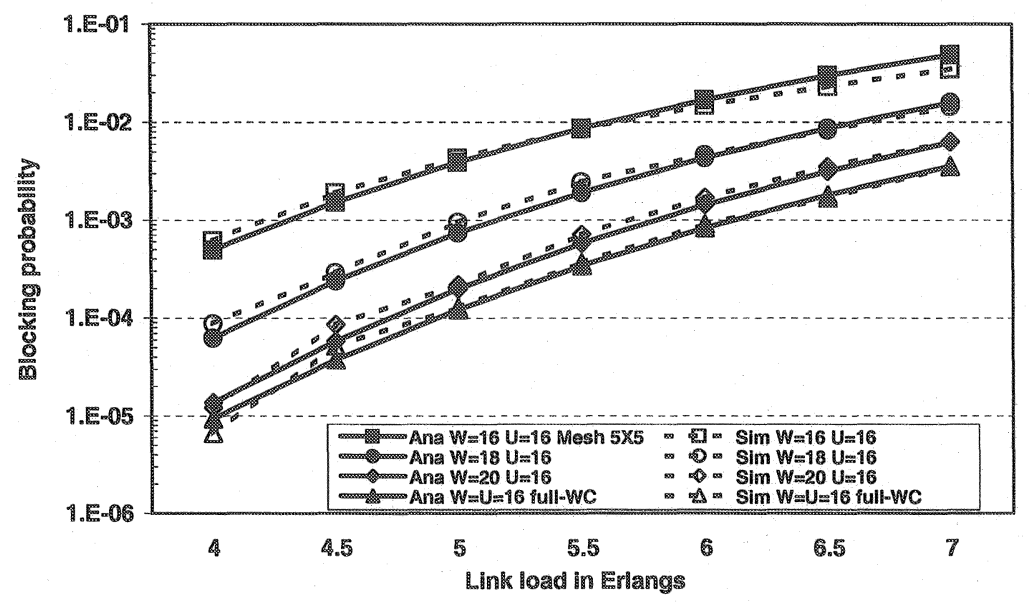

Figure 5. Blocking probability versus the link offered load for a bidirectional $5 \times 5$ MeshTorus network.

It is observed that the blocking performance of networks with higher correlation ratio will benefit more compared to those with lower correlation with the extra wavelengths. From the graphs, it can be seen that the addition of four extra wavelengths results in almost the same blocking performance as the case with full-wavelength conversion in ring networks as compared to mesh-torus. This effect can be significant with the increase in the network size. This effect is also due to the fact that wavelength converters do not result in a drastic reduction in the blocking performance for networks with higher correlation ratio $[9,10]$. As most of the existing real-life networks have sparse connectivity, therefore having a high link load correlation, the approach of providing extra wavelengths is attractive compared to employing full-wavelength conversion.

\section{Conclusion}

We consider the power budget scenario in optical networks when the total number of usable wavelengths in a fiber is limited to a certain maximum number due to power considerations. The total number of available wavelengths in the fiber can be more then the maximum usable number, referred to as the wavelength usage constraint. In this paper, we develop an analytical model for evaluating the blocking performance of WDM optical networks with wavelength usage constraint. The analytical model is shown to be accurate by comparing the results with that of the simulation for two different network topologies that have high and low link load correlation. It is observed that with an increase of few extra wavelengths in the fiber, the blocking performance is similar to that when full-wavelength conversion is employed. Thus employing 
extra wavelengths in practical networks appears to be an attractive alternative compared to employing full-wavelength conversion even in the presence of power budget constraints.

In this paper, we have developed an analytical model with the assumption of random wavelength assignment. It would be an interesting future work to study the effects of other wavelength assignment algorithms in the context of wavelength usage constraint.

\section{References}

[1] Barry, R. and P. Humblet: 1996, 'Models of blocking probability in all-optical networks with and without wavelength changers'. IEEE Journal of Selected Areas in Communications 14(5), 858-867.

[2] Bertsekas, D. and R. Gallager: 1992, Data Networks. Printice Hall.

[3] Birman, A.: 1995, 'Computing approximate blocking probabilities for a class of alloptical networks'. In: Proceedings of IEEE INFOCOM'95. pp. 651-658.

[4] Kovacevic, M. and S. Acampora: 1995, 'On wavelength translation in all-optical networks'. In: Proceedings of IEEE INFOCOM'95. pp. 413-422.

[5] Kovacevic, M. and S. Acampora: 1996, 'Benefits of wavelength translation in all-optical clear-channel networks'. IEEE Journal of Selected Areas in Communications 14(5), 868880.

[6] Lee, K. C. and V. O. K. Li: 1993, 'A wavelength-convertible optical network'. Journal of Lightwave Technology 11(5), 962-970.

[7] Li, L. and A. Somani: 1999, 'A new analytical model for multi-fiber WDM networks'. In: Proceedings of the Global Telecommunications Conference, GLOBECOM'99, Vol. 1B. pp. 1007-1011.

[8] Ramaswami, R. and G. Sasaki: 1998, 'Multiwavelength optical networks with limited wavelength conversion'. IEEE/ACM Transactions on Networking 6(6), 744-754.

[9] Srinivasan, R. and A. K. Somani: 2001, 'A generalized framework for analyzing timespace switched optical networks'. In: Proceedings of IEEE INFOCOM 2001. pp. 179188.

[10] Subramaniam, S., M. Azizoglu, and A. Somani: 1996, 'All-optical networks with sparsewavelength conversion'. IEEE/ACM Transactions on Networking 4(4), 544-557.

[11] Tripathi, T. and K. Sivarajan: 1999, 'Computing approximate blocking probabilities in wavelength-routed all-optical networks with limited-range wavelength conversion'. In: Proceedings of IEEE INFOCOM'99, Vol. 1. pp. 329-336.

[12] Wauters, N. and P. Demeester: 1997, 'Wavelength conversion in optical multi-wavelength multi-fiber transport networks'. International Journal of Optoelectronics $11(1), 53-70$.

[13] Yates, J., J. Lacey, and D. Everitt: 1996, 'Blocking in multiwavelength TDM networks'. In: 4th International Conference on Telecommunication Systems, Modeling, and Analysis. pp. 535-541. 